\title{
Q \& A with the man who can stop cervical cancer in its tracks
}

ichard Schlegel is a pioneer in human papillomavirus (HPV) research. Schlegel, chairman of the Department of Pathology at Georgetown University Medical Center, has long studied the protein shell surrounding HPV. His work led to the development of a vaccine called Cervarix, currently in clinical trials. The trouble with Cervarix is that it is solely preventative, not therapeutic, and is difficult to transport because it must be kept frozen. Now, with the help of a new award from the Bill \& Melinda Gates Foundation and the NIH, Schlegel's stellar team has 3.5 million dollars to create the next generation HPV vaccine, which will be both preventative and therapeutic, can be produced inexpensively in bacteria, and can be shipped in powder form and reconstituted simply with water to be used in developing countries all over the world. Schlegel spoke to the JCI about this award, the link between HPV and cervical cancer, and what he hopes to accomplish.

JCI: What does your lab study?

Schlegel: The basic biology of papillomaviruses and their role in human cancer. We use molecular, cellular, and animal models to study the viral genes that alter growth of human cells and facilitate the development of cancer. We also pursue translational research aimed at developing vaccines to prevent and treat papillomavirus infections as well as drug therapies to treat these infections.

JCI: Why is HPV such a big health problem?

Schlegel: HPV generates virtually all cervical cancers in women, and nearly 250,000 women die from this cancer each year. There are no therapeutic drugs which specifically target HPV, so the current approach taken by pharmaceutical companies is to develop and market a prophylactic vaccine that can be administered early in life and prevent HPV infection and the development of cervical cancer.

JCI: What is the precise relationship between HPV and cervical cancer?

Schlegel: More than $99 \%$ of human cervical cancers contain and express the oncogenes of the papillomavirus. There are two oncogenes in HPV that facilitate the development of cancer, E6 and E7. However, while E6 and E7 oncogenes are sufficient to immortalize human cells in vitro, they don't induce immediate conversion to the tumorigenic state. It appears that additional genetic changes in the host cell must occur for an HPV-infected cell to become fully transformed.

JCI: Why is HPV so prevalent among women?

Schlegel: HPV is transmitted sexually and infects both men and women. While there appears to be no preferential infection of women, women are prone to developing cancer following infection, which most likely reflects differences in the tissues that are infected. The "transition zone" of the cervix is the most susceptible to cancer.

JCI: How did you get interested in this field?

Schlegel: I became interested in viral oncology when I was working as a postdoctoral fellow with Tom Benjamin at Harvard University. Afterward, I moved to the NIH and worked with Peter Howley on the bovine papillomavirus, and soon it was discovered that there were human papillomaviruses which were associated with cervical cancer.

JCI: How were you selected for this award?

Schlegel: I lead one of four research centers collaborating to develop a prophylactic and therapeutic vaccine for the human papillomaviruses and cervical cancer. The research groups are based in the University of Colorado Health Sciences Center, the German National Cancer Center, the Ludwig Cancer Center in Brazil, and Georgetown. The Gates Foundation announced a competition for researchers to respond to critical issues of global health. They received 1,700 proposals and used a panel to assess the scientific basis and health impact of the proposals.

JCI: How do you coordinate this international research effort?

Schlegel: The research is administratively coordinated at the University of Colorado by Dr. Robert Garcea who serves as the principal investigator. I have worked with several of the investigators before, and we have been very successful using the internet, phone calls, and meetings to coordinate our efforts. We also have travel support as part of our grant so we can meet regularly.

JCI: What sort of timeline do you envision with regards to creating a safe and effective HPV vaccine?

Schlegel: A safe, effective, prophylactic vaccine will be marketed within 1-2 years. However, the second-generation prophylactic/therapeutic vaccine we are working on would not be available for another $7-10$

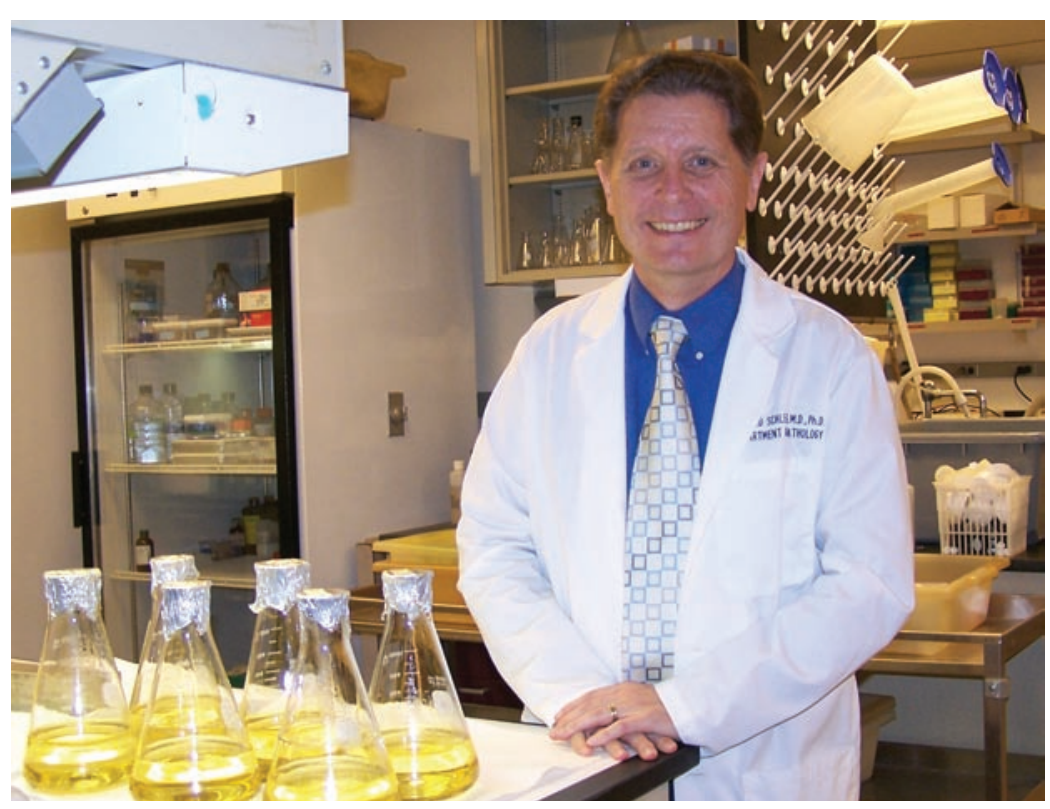

Richard Schlegel in his laboratory, where his work on HPV could lead to the first therapeutic vaccine for cervical cancer. 
years, assuming that it was highly effective. JCI: What is your typical day like?

Schlegel: I usually get to work around 7 AM so I have about 2 hours to read, write, and think before the rest of the lab arrives. After that, I mainly respond to research and administrative issues. In general, I work after dinner on the internet to screen the literature relevant to our research.

JCI: What do you consider to be your greatest scientific accomplishment?
Schlegel: From a clinical perspective, I think my most significant accomplishment has been contributing to the development of a vaccine that will have a significant positive impact on world health. From a basic science standpoint, I have been most satisfied with our studies of the mechanism by which the E5 oncoprotein alters cell growth by modifying the activity of the cellular V-ATPase.

JCI: What about your greatest life accomplishment?
Schlegel: Overall, my greatest satisfaction is having sufficient time to raise and enjoy a wonderful family.

JCI: By the time this money runs out, what do you hope to have achieved?

Schlegel: Our greatest excitement would come if we could produce an inexpensive, stable HPV vaccine that could be used on patients before and after infection.

\section{Stacie Bloom}

\section{Nucleic acid sequence data turns $100,000,000,000$ and looks to the future}

$T_{\text {he }}$ Nucleotide Sequence Database Collaboration (INSDC) - the European Molecular Biology Laboratory (EMBL) Bank, GenBank, and the DNA Data Bank of Japan (DDBJ) - have reached a milestone. Owing in large part to their daily exchange policies, these public databases for DNA and RNA sequences have reached 100 gigabases of information.

These 100,000,000,000 bases of genetic code, collected since 1982, comprise over 55 million sequence entries from more than 200,000 different organisms. This collaborative effort ensures that information gleaned from molecular biology and genetic research is placed in the public domain where the scientific community can use it to push science forward.

"Today's nucleotide sequence databases allow researchers to share completed genomes, the genetic makeup of entire ecosystems, and sequences associated with patents," said David Lipman, director of the National Center for Biotechnology Information. "The INSDC has realized the vision of the researchers who initiated the sequence database projects by making the global sharing of nucleotide sequence information possible."

The repositories got started in the 1970s, when researchers suggested a public storehouse be made available for the massive amounts of genetic code sequence information that were being generated. Two of the databases - the EMBL Data Library and GenBank - were launched in the early 1980s. The European Bioinformatics Institute (EBI) manages the EMBL database, while GenBank is the NIH's National Center for Biotechnology Information genetic sequence database.

Both EMBL and GenBank offer an anno- tated collection of all publicly available DNA sequences, and they were formed as nonprofit entities that collaborated from the beginning. By 1987, the INSDC was formed and included a third collaborator - DDBJ, launched at the National Institute of Genetics in Mishima. DDBJ is also an international nucleotide sequence database freely accessible online.

Early on, staffers searched published journal articles for sequence data and entered it manually into the repository. But times have changed, and the modern sequencing centers at universities today have come a long way. New automated technology, robotics, and bioinformatics, combined with decreased cost, have fostered faster data collection.

"The technology has come so fast that it blows my mind," said Richard Wilson, director of the Genome Sequencing Center at Washington University School of Medicine. Wilson explained that in the mid-1980s his center was able to turn out several hundred bases of sequence per month, while today they are generating about 4.2 billion.

A boost to the number of collected sequences is also due to the National Human Genome Research Institute (NHGRI) at the NIH, which now provides genome-sequencing grants. These funds support research aimed at sequencing a human-sized genome at a cost 100 times lower than is possible now - it presently costs nearly 10 million dollars to sequence the 3 billion base pairs of DNA found in humans. The immediate goal of the NHGRI is to lower the cost of these projects by tens of thousands of dollars in order to allow scientists to sequence genomes of human subjects involved in studies to find genes relevant for disease. The longer-term

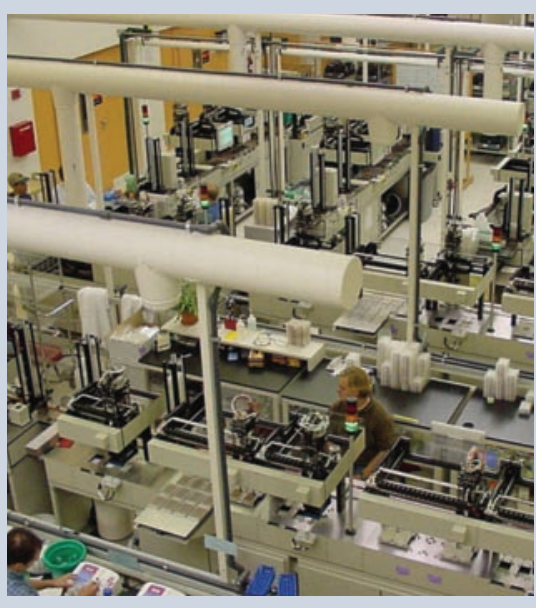

Large-Scale Sequencing Center at the Whitehead Institute. Today, novel automation technologies and computational packages for genome analysis are available to step up data collection. Photo courtesy of the $\mathrm{NIH}$.

NHGRI goal is to reduce whole-genome sequencing to only 1,000 dollars so that this process can be used in routine medical tests and allow physicians to tailor diagnosis, prevention, and treatment to a patient's individual genetic makeup.

So far, the genetic information available has taught us a lot about the evolutionary relationships among different species. But according to Richard Gibbs, director of the Human Genome Sequencing Center at Baylor College of Medicine, "This will pale into insignificance once we find the full repertoire of alleles that cause different human genetic disease." These are the tools, he explained, that would unlock our understanding of gene function and ultimately provide diagnostic and prognostic indicators. The benefit to human health will be immeasurable.

\section{SB}

